\title{
Retraction Note to: Towards End-to-End DNN-Based Identification of Individual Manta Rays from Sparse Imagery
}

Tuana Celik, Benjamin Hughes, and Tilo Burghardt

\author{
Retraction Note to: \\ Chapter "Towards End-to-End DNN-Based Identification \\ of Individual Manta Rays from Sparse Imagery" \\ in: R. Vera-Rodriguez et al. (Eds.): Progress in Pattern \\ Recognition, Image Analysis, Computer Vision, \\ and Applications, LNCS 11401, \\ https://doi.org/10.1007/978-3-030-13469-3_75
}

The authors have retracted this chapter [1] because the results described in this work are based on an incorrect data set, which means that the experimental results cannot be reproduced. All authors agree with this retraction.

[1] Celik, T., Hughes, B., Burghardt, T.: Towards End-to-End DNN-Based Identification of Individual Manta Rays from Sparse Imagery. In: Vera-Rodriguez, R., Fierrez, J., Morales, A. (eds.) CIARP 2018. LNCS, vol. 11401, pp. 645-653. Springer, Cham (2019). https://doi.org/10.1007/978-3-030-13469-3_75 